\title{
Operationalizing Neuroimaging for Disorders of Consciousness in the Canadian Context
}

\author{
K. Julia Kaal, Mark Harrison, David G.T. Whitehurst, Andrea Townson, \\ Derry Dance, A. Jon Stoessl, Judy Illes
}

Keywords: Brain injury-traumatic; Functional neuroimaging, Health services research, Health economics doi:10.1017/cjn.2018.316

Can J Neurol Sci. 2018; 45: 633-635

Traumatic brain injury is a significant cause of death and disability affecting tens of thousands of Canadians each year. A small but significant number of patients do not regain full consciousness and remain in a vegetative state (VS), also referred to as unresponsive wakefulness syndrome (UWS). The adequacy of conventional assessments of patients with such disorders of consciousness (DoC) has been challenged because clinical examination can underestimate the extent of patients' residual cognitive function. ${ }^{1}$ The use of functional magnetic resonance imaging (fMRI) to detect signals interpreted as consciousness has yielded substantial attention and debate for the neurological sciences, and Lee et al. report wide-ranging optimism among health and legal experts for clinically operationalizing fMRI for patients with DoC. ${ }^{1,2}$

Evidence of fMRI signals interpreted as consciousness in VS/ UWS patients ${ }^{3}$ highlights the need to reconsider levels of cortical activity in DoC, and to acknowledge the personhood of these patients. Here, we offer a view on how the use of fMRI as an adjunct diagnostic method to clinical examination might feasibly change the clinical care pathway for these individuals.

We begin with the premise that a standardized clinical care pathway is a priority for two reasons: (i) it ensures that the condition of beneficence is met, and (ii) it serves as a foundation for future economic modeling to estimate the value of diagnostic or therapeutic interventions of this kind within the health care system. The following assumptions guide our approach:

1. There is clinical utility of fMRI for single-patient diagnostics. ${ }^{4}$

2. The 30-day mark signifies the point when the term "persistent VS/UWS" is often used for the first time. ${ }^{5,6}$ This also coincides with the time at which families and care providers anticipate a prognosis, which provides information that will be used to make long-term care decisions.

3. The presence of fMRI signals interpreted as consciousness may serve as the basis to re-classify a patient as "positive signal minimally conscious state (MCS)."

4. Persistent reduced levels of consciousness at the 1-year time point mark a transition in classification from persistent VS/UWS to permanent VS/UWS. ${ }^{5}$

5. Health care resource requirements are most intensive during the first 12 months post-injury. ${ }^{5}$
6. Compared with the first 12 months, care costs over the 13to 24-month period may provide a more accurate annual estimate to extrapolate lifetime costs because diagnosis and prognosis are established and the care trajectory is more predictable. $^{5}$

Figure 1 illustrates the hypothetical care pathway. It considers newly brain-injured patients who do not regain full consciousness, that is, patients who present with persistent reduced levels of consciousness with no apparent treatable cause. The pathway distinguishes between patients in VS/UWS and MCS based on clinical diagnosis. Only patients presenting clinically with VS/ UWS undergo fMRI at the 30-day mark. The fMRI may show the presence of signals interpreted as consciousness, equivocal presence of signals, or no presence of signals. Those with no or equivocal presence of signals or equivocal presence of signals at the 30-day mark are reassessed using fMRI at the 90-day mark to identify whether there has been any change in the presence of signals interpreted as consciousness. In the event of another negative or equivocal result at 90 days, patients undergo a further fMRI study at 12 months. The presence of signals interpreted as consciousness at the 90-day or 12-month mark may serve as the basis to re-classify patients as "positive signal MCS." Patients with consistent negative or equivocal results would not be reclassified, and a diagnosis of permanent VS/UWS would be made at 12 months. ${ }^{5}$

From the Faculty of Pharmaceutical Sciences, University of British Columbia, Vancouver, British Columbia, Canada (KJK, MH); School of Population and Public Health, University of British Columbia, Vancouver, British Columbia, Canada (KJK); Centre for Health Evaluation and Outcome Sciences, St Paul's Hospital, Vancouver, British Columbia, Canada (MH); Faculty of Health Sciences, Simon Fraser University, Vancouver, British Columbia, Canada (DGTW); Centre for Clinical Epidemiology and Evaluation, Vancouver Coastal Health Research Institute, Vancouver, British Columbia, Canada (DGTW); Department of Medicine, Division of Physical Medicine and Rehabilitation, University of British Columbia, Vancouver, British Columbia, Canada (AT, DD); Department of Medicine, Division of Neurology, University of British Columbia, Vancouver, British Columbia, Canada (AJS); Neuroethics Canada, Department of Medicine, Division of Neurology, University of British Columbia, Vancouver, British Columbia, Canada (JI).

Received December 13, 2017. Final Revisions Submitted June 6, 2018. Date of ACCEPTANCE JuNE 16, 2018.

Correspondence to: Judy Illes, Professor of Neurology, Department of Medicine and Director, Neuroethics Canada, The University of British Columbia, 2211 Wesbrook Mall, Koerner S124, Vancouver, BC, Canada V6T 2B5. Email: jilles@mail.ubc.ca 


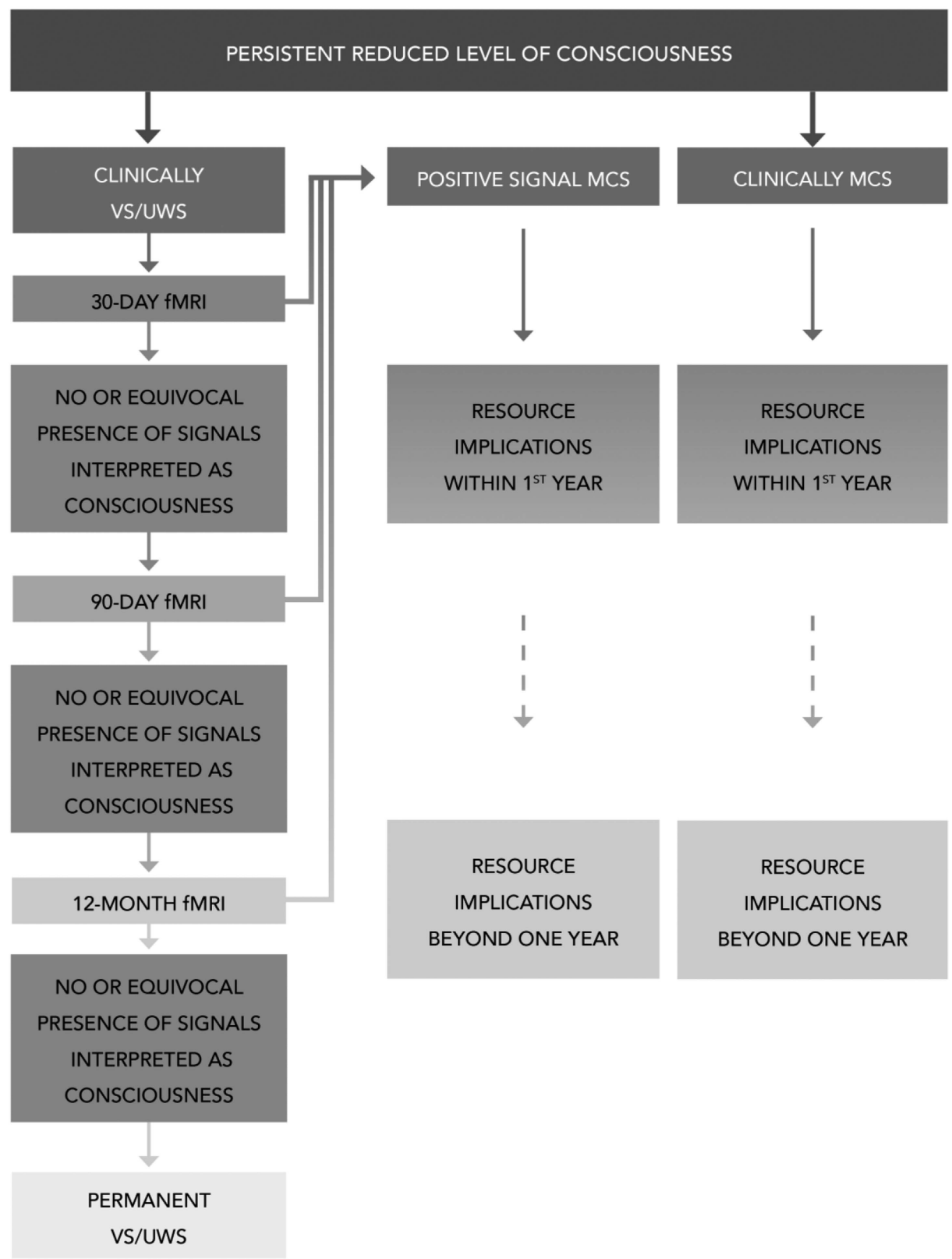

Figure 1: Care pathway for functional magnetic resonance imaging (fMRI) assessment of patients with disorders of consciousness. MCS = minimally conscious state; UWS = unresponsive wakefulness syndrome; $V S=$ vegetative state.

Table 1 summarises potential implications of the different fMRI outcomes with regard to patient prognosis, the need for continued assessment, health care resource use, and ethical and moral considerations for the patient, the family, and the care team. Table 1 also highlights current uncertainties associated with the respective outcomes of the fMRI. For example, factors associated with an equivocal fMRI outcome will probably lead to the retention of patients in more resource-intense settings, such as acute or rehabilitation facilities. This eventuality may also increase friction between families and care teams, and affect medical management plans, especially in the face of complex medical decisions, such as those associated with invasive procedures to sustain life. Although we identify the initial 12 -month period as the most resource-intensive, precise estimates of the cost of resource use beyond the fMRI evaluation itself are uncertain. It may be that changes in the diagnosis within the initial 12-month period are more predictive of resource implications in subsequent periods.

From an economic perspective, any differences in resource use and health care costs arising from the implementation of fMRI in clinical practice would need to be considered in the context of the value of any measurable change in outcomes. Without the ability to quantify outcomes in terms of the impact on the quality of life of patients, and perhaps the quality of life of individuals close to the patients, it is not possible to state with any certainty whether allocating scarce health care resources to the operationalization of fMRI would meet a decision-makers definition of "value for 
Table 1: Possible outcomes of functional magnetic resonance imaging (fMRI) as an adjunct diagnostic examination for patients with vegetative state/unresponsive wakefulness syndrome (VS/UWS) between 30 days and 12 months

\begin{tabular}{|c|c|c|c|}
\hline & Presence of signals interpreted as consciousness & $\begin{array}{c}\text { Equivocal presence of signals interpreted as } \\
\text { consciousness }\end{array}$ & $\begin{array}{l}\text { No presence of signals } \\
\text { interpreted as consciousness }\end{array}$ \\
\hline Prognosis & $\begin{array}{l}\text { More likely to improve to clinical MCS or possibly } \\
\text { emerge from MCS }\end{array}$ & Unclear prognosis & Guarded prognosis \\
\hline Assessment & $\begin{array}{l}\text { Re-evaluate for positive clinical changes } \\
\text { If individual changes, intermittent clinical re- } \\
\text { evaluation may be warranted }\end{array}$ & $\begin{array}{l}\text { Continue current standard care/evaluation } \\
\text { Anticipate more congruent imaging and clinical } \\
\text { presentation over time }\end{array}$ & $\begin{array}{l}\text { Continue current standard care/ } \\
\text { evaluation } \\
\text { Anticipate congruent imaging } \\
\text { and clinical presentation over } \\
\text { time }\end{array}$ \\
\hline Resource implications & $\begin{array}{l}\text { No change in routine care. Modest increase in resources } \\
\text { for re-assessment, for example, therapists, rehab } \\
\text { medicine } \\
\text { If improving clinically (i.e., emerging from MCS), } \\
\text { significant increase in rehabilitation resources }\end{array}$ & $\begin{array}{l}\text { No change in routine care. May facilitate discussion } \\
\text { and planning of longer-term care arrangements } \\
\text { Equivocal fMRI may increase re-evaluation } \\
\text { resources }\end{array}$ & $\begin{array}{l}\text { No change in routine care. } \\
\text { Consistent information useful } \\
\text { in facilitating care planning }\end{array}$ \\
\hline \multirow[t]{6}{*}{ Ethical/moral considerations } & Patient & Patient & Patient \\
\hline & $\begin{array}{l}\text { Patient preferences if known to surrogate decision- } \\
\text { maker, quality of life, context support and resources, } \\
\text { potential for limited communication }\end{array}$ & $\begin{array}{l}\text { Wait to see whether autonomy resumes and becomes } \\
\text { exercisable } \\
\text { Re-evaluate }\end{array}$ & $\begin{array}{l}\text { No change in status quo } \\
\text { Re-evaluate }\end{array}$ \\
\hline & Family/others & Family/others & Family/others \\
\hline & $\begin{array}{l}\text { Patients' best interests, uncertainty for surrogate in } \\
\text { decision-making if limited clinical progression }\end{array}$ & $\begin{array}{l}\text { Patients' best interests, uncertainty for surrogate in } \\
\text { decision-making, particularly where clinical } \\
\text { presentation and imaging are discrepant }\end{array}$ & $\begin{array}{l}\text { Likely to provide useful direction } \\
\text { in decision-making }\end{array}$ \\
\hline & Care team & Care team & Care team \\
\hline & $\begin{array}{l}\text { Patients' best interests, resource allocation and waiting } \\
\text { times, professional uncertainties/conflicts if limited } \\
\text { clinical progression }\end{array}$ & $\begin{array}{l}\text { Increasingly complicated rather than ameliorated } \\
\text { patient care and referral pathway, and family } \\
\text { counseling scenario }\end{array}$ & $\begin{array}{l}\text { Clear diagnostic picture; } \\
\text { consistent health care planning }\end{array}$ \\
\hline
\end{tabular}

money." The assessment of quality of life of patients with DoC remains a considerable challenge and an area that requires further research.

For the purposes of this work - a starting point in the conversation-we recognize that the presented pathways and associated discussion cannot capture fully the complexity, heterogeneous physical requirements, and ethical duties of caring for patients with traumatic brain injury. The challenges are exacerbated by the absence of any pre-existing, standardized care pathway against which to compare. We are also not concerned with a statistical definition of what constitutes the presence of signals interpreted as consciousness or the particular type of imagining (e.g., resting state fMRI functional connectivity, regional brain activity, or task/command-evoked activity) to be used. Instead, we seek to highlight the multi-dimensionality of fMRI outcomes with regard to patient prognosis, continued assessment, health care resource use, ethical and moral implications, and the extent of research still needed before this technology can be fully considered for translation into the clinical setting. Nonetheless, this is one more contribution to the continued pursuit to understand human consciousness after neurotrauma, in an effort to eventually apply that knowledge to the clinical care of affected patients for whom hope and rights have been historically neglected.

\section{ACKNOWLEDGMENTS}

JI is Canada Research Chair in Neuroethics. This work was supported by Canadian Institutes of Health Research (CIHR) \#EOG-120257 (JI) and the Vancouver Coastal Health Research Institute.

\section{Disclosure}

KJK, MH, DGTW, AT, DD, and AJS have nothing to disclose. JI reports grants from Canadian Institutes of Health Research (CIHR \#EOG-120257), the Vancouver Coastal Health Society, and Canada Research Chairs program, during the conduct of the study.

\section{Statement of Authorship}

JI conceived the idea for the project. All authors provided critical feedback and helped to shape the research. KJK took the lead in writing the manuscript.

\section{REFERENCES}

1. Rodriguez Moreno D, Schiff ND, Giacino J, Kalmar K, Hirsch J. A network approach to assessing cognition in disorders of consciousness. Neurology. 2010;75(21):1871-8.

2. Lee G, Byram AC, Owen AM, et al. Canadian perspectives on the clinical actionability of neuroimaging in disorders of consciousness. Can J Neurol Sci. 2015;42(2):96-105.

3. Owen AM, Coleman MR, Boly M, Davis MH, Laureys S, Pickard JD. Detecting awareness in the vegetative state. Science. 2006;313(5792):1402.

4. Demertzi A, Antonopoulos G, Heine L, et al. Intrinsic functional connectivity differentiates minimally conscious from unresponsive patients. Brain. 2015;138(9):2619-31.

5. Multi-Society Task Force on PVS. Medical aspects of the persistent vegetative state. N Engl J Med 1994;330(21):1499г508.

6. Prolonged disorders of consciousness: national clinical guidelines. RCP London; 2015. Available at: https://www.rcplondon.ac.uk/ guidelines-policy/prolonged-disorders-consciousness-nationalclinical-guidelines. 